ARTÍCULO ORIGINAL

Rev Colombiana Cienc Anim 2017; 9(1):73-80.

\title{
Circunferencia escrotal y parámetros morfométricos en machos Bubalus bubalis de la raza Murrah
}

\section{Scrotal circumference and morphometric in males Bubalus bubalis of the race Murrah}

\author{
Espitia P, Amado ${ }^{1}$ M.Sc, Montes V, Donicer ${ }^{1}$ Ph.D, Hernandez M, Enelida ${ }^{1,2}$ Zoot, \\ Sfeir B, Hernando ${ }^{1,2}$ Zoot.
}

${ }^{1}$ Universidad de Sucre, Facultad de Ciencias Agropecuarias, Departamento de Zootecnia, Sincelejo, Colombia. ${ }^{2}$ Grupo de Investigación en Mejoramiento y Reproducción Animal.

\section{Keywords:}

Buffalo;

correlation; morphometry;

reproduction

\begin{abstract}
In the present study the scrotal circumference of male buffaloes (Bubalus bubalis, Murrah race) was characterized and its relationship with other morphometric parameters was defined. The study was realized at "El Mosquito" farm, in San Marcos municipality in Sucre, Colombia. Fourteen (14) male buffaloes were taken from their weaning to twenty months old. These animals were subjected to testis measures (SC, testis longitude), body weight, and croup height, every twenty-eight days. To establish SC of the group under study, descriptive statistics was used, mean and standard deviation. In the same way, simple correlation analysis between SC with age, weight, croup height and testicular length (right and left). SC increased during experimental monitoring from $12,4 \pm 1,0 \mathrm{~cm}$ to $20,2 \pm 1,4 \mathrm{~cm}$. The weight ranged from $162,1 \pm 24,9 \mathrm{~kg}$ to $323,8 \pm 39,8 \mathrm{~kg}$. LTD fluctuated from $4,0 \pm 0,7 \mathrm{~cm}$ to $7,4 \pm 0,7$ $\mathrm{cm}$. LTI began from $4,2 \pm 0,5 \mathrm{~cm}$ and finished in $6,9 \pm 0,6 \mathrm{~cm}$ and height rump at 10 months, was $104 \pm 4,4 \pm 3,4 \mathrm{~cm}$ to $118 \mathrm{~cm}$ at 20 months old male buffaloes studied. In conclusion, correlations between $\mathrm{CE}$ and age, weight, right and left testis length height and height haunch were: $r=0,84 ; 0,90 ; 0,93 ; 0.91$, and 0,71 respectively. It's concluded growth to SC was not uniform, also a high correlation was found between SC and morphometric parameters studied.
\end{abstract}

\section{Resumen}

Objetivo: Caracterizar la circunferencia escrotal (CE) y su relación con otros parámetros morfometricos en machos bufalinos (Bubalus bubalis) de la raza Murrah en la Hacienda Mosquito, Municipio de San Marcos - Sucre - Colombia. Metodología: Se tomaron 14 machos a partir del destete hasta los 20 meses de edad, se les realizaron medidas testiculares (CE, longitud testicular), peso corporal y alzada al anca, cada 28 días. Para establecer la CE del grupo en estudio se utilizó estadística descriptiva, media y desviación estándar. Igualmente análisis de correlaciones simples entre CE con edad, peso, alzada al anca y longitud testicular (derecha e izquierda). Resultados: La CE aumentó durante el seguimiento experimental de 12,4 $\pm 1,0 \mathrm{~cm}$ a 20,2 $\pm 1,4 \mathrm{~cm}$. El peso osciló de 162,1 $\pm 24,9 \mathrm{~kg}$ a $323,8 \pm 39,8 \mathrm{~kg}$. La LTD fluctúo de $4,0 \pm 0,7 \mathrm{~cm}$ a $7,4 \pm 0,7 \mathrm{~cm}$. La LTI inició con $4,2 \pm 0,5 \mathrm{~cm}$ y culminó con $6,9 \pm 0,6 \mathrm{~cm}$ y la alzada al anca a los 10 meses fue de $104 \pm 4,4 \mathrm{~cm}$ a $118 \pm 3,4 \mathrm{~cm}$ a los 20 meses de edad de los animales estudiados. Las correlaciones entre CE con edad, peso, longitud testicular derecha e izquierda y alzada al anca fueron: $r=0,4,0,90 ; 0,93 ; 0,91$, y 0,71 respectivamente. Se concluye, que el crecimiento de la CE no fue uniforme, además se encontró una alta correlación entre la CE y los parámetros morfometricos estudiados. 


\section{Introducción}

La circunferencia escrotal (CE), es un indicador muy importante de fertilidad de los machos, partiendo de la base que el mayor progreso genético se logra a través de la selección por este carácter, su heredabilidad permite mejorar parámetros reproductivos en machos y hembras (CORBET et al., 2012 RAMÍREZ et al., 2016). La CE se define como el tamaño testicular expresado en $\mathrm{cm}$, y se relaciona con la cantidad de epitelio seminífero presente en el parénquima testicular. Es un parámetro utilizado con frecuencia en programas de mejoramiento genético animal, debido a su fácil medición, alta repetíbilidad y heredabilidad moderada a alta. (BOURDON Y BBRINKS, 1986; TERAKADO et al., 2015; IRANO et al., 2016).Por estas razones es considerado un carácter interesante al seleccionar machos en un sistema de producción animal.

Las relaciones de las medidas testiculares con la producción de semen y la fertilidad en los machos, han demostrado que la CE está significativamente correlacionada con la concentración de espermatozoides por eyaculado, el volumen escrotal y el peso corporal (RAMÍREZ et al., 2016; ALBADRY et al., 2016); por otro lado, NEMA y KODAGALI (1994) demostró que en búfalos, CE más amplia producen semen de mejor calidad y morfología.

En sistemas ganaderos extensivos, la función reproductiva del macho es de suma importancia, ya que un solo macho es capaz de cubrir a un gran número de hembras, por tanto, deficiencias en su capacidad reproductiva altera la fertilidad en mayor medida en que lo haría una sola hembra con problemas reproductivos (MATEOS, 1992).

Algunos investigadores (VALE et al., 2004), han indicado que en búfalo, los parámetros morfometricos de CE, han sido poco estudiados, siendo este, un parámetro importante para la evaluación andrológica. El peso corporal y la CE son dos parámetros importantes utilizados en la evaluación de la capacidad reproductiva de los machos bovinos y bufalinos (SILVA et al., 2012; Da LUZ et al., 2012; PÉREZ et al., 2014).

En los búfalos machos es posible que el desarrollo corporal y del aparato genital de esta especie puede alcanzar su pleno desarrollo en una forma similar a los bovinos, dependiendo de la presión de selección, del manejo de los animales desde el nacimiento, pasando por el destete y continuando por las diferentes fases de producción (VALE, 2011). La literatura disponible del tamaño de los testículos en el búfalo, relacionada con algunos parámetros morfometrico de estructura y peso es limitada (AHMAD et al., 2010), el objetivo de este estudio fue: Caracterizar la circunferencia escrotal
(CE) y su relación con otros parámetros morfometricos en machos Bubalus bubalis de la raza Murrah en la Hacienda Mosquito, Municipio de San Marcos Sucre - Colombia. Esta información, contribuirá con el conocimiento zootécnico de los sistemas ganaderos bufalinos criados en Colombia.

\section{Metodología}

El presente estudio se llevó a cabo en las Haciendas San Juan de Luis y Mosquito. La Hacienda San Juan de Luis se encuentra ubicada en el corregimiento el Viajano, jurisdicción del Municipio de Sahagún, Departamento de Córdoba Colombia ( $8^{\circ} 37^{\prime} 45.9^{\prime \prime}$ de latitud norte y $-75^{\circ} 28^{\prime} 39.58^{\prime \prime}$ de longitud occidental), a $124 \mathrm{msnm}$, con temperatura promedio de $28^{\circ} \mathrm{C}$ (GOOGLE EARTH, 2016). La Hacienda Mosquito, está ubicada en el Municipio de San Marcos, Departamento de Sucre $\left(8^{\circ} 39^{\prime} 40^{\prime \prime}\right.$ de latitud Norte y $75^{\circ} 08^{\prime} 05^{\prime \prime}$ de longitud Occidental), a $28 \mathrm{msnm}$, con temperatura media de $28.4^{\circ} \mathrm{C}$ y precipitación anual de $1124 \mathrm{~mm}$ (IGAG, 2010).

Fue utilizada la información correspondiente a 14 machos bufalinos de la raza Murrah desde el destete (10 meses) hasta los 20 meses de edad. Las medidas consideradas para la evaluación fueron: Alzada a grupa, medida en $\mathrm{cm}$ desde el suelo hasta la tuberosidad coxal, Peso Corporal (PC), cuantificado con (BASCULA PROMETALICOS ${ }^{\circledR}$ ) balanza mecánica; Circunferencia escrotal (CE),siendo medida a nivel del diámetro mayor de ambos testículos con un escrotímetro, graduado en cm (PEREZ et al ., 2014) .

La longitud testicular derecha e izquierda, fue tomada con un calibrador Pie de Rey. Para lograr este propósito se delimitó la cabeza y cola del epidídimo, los testículos fueron bajados con firmeza hasta el fondo de la bolsa escrotal, determinando así la longitud de cada uno.

El estudio se inició en la hacienda San Juan de Luis, los dos primeros meses. La alimentación de los semovientes durante este periodo, se basó en su totalidad en pasturas predominantes en la región, como el pasto colosuana (Bothriochloa pertusa), aunque el experimento se inició en época de lluvias se observó poca oferta forrajera. Transcurridos dos meses, los animales fueron trasladados a la hacienda Mosquitos, manteniéndose en pastoreo continuo y extensivo en suelos planos y mal drenados, en praderas de pastos naturales como: Antephora hermafrodita (canutillo), Leerxia hexandra (Lambe lambe) y Paspalum fasciculatum (Gramalote). También se encontraron arvenses, como Artemisia vulgaris, (Altamisa), Malachra alceifolia, (Malva), Rhynchospora nervosa, (Cortadera), empalizadas y algunas marantáceas como calathea lútea (bijao), que se entrecruzan con caños, y bosques naturales. 
Se realizó estadística descriptiva (media aritmética y desviación estándar) para las variables evaluadas, por medio del programa estadístico STATGRAPHICS plus versión 5.1. Se realizaron análisis de regresión en función de la CE con todos los parámetros evaluados. Se determinaron los coeficientes de correlación de Pearson para las paramétricas: circunferencia escrotal, edad, peso, longitud testicular (derecha- izquierda) y alzada a grupa. De acuerdo con su normalidad, los coeficientes de correlación entre las variables fueron validados por un segundo método, definido por SAMPAIO, 2002.

\section{Resultados}

Circunferencia escrotal en Búfalos Murrah (10-20 meses). Los resultados de la caracterización de la CE en búfalos de la raza Murrah desde el destete (10 meses) hasta los 20 meses de edad se presentan en la Tabla 1 , sus valores oscilando entre $12,4 \pm 1,0 \mathrm{~cm}$ y $20,2 \pm 1,4 \mathrm{~cm}$ en el inicio y final del periodo evaluado respectivamente; es decir, tuvo un aumento de $7,8 \mathrm{~cm}$ a lo largo del estudio (10 meses). En la figura 1, se puede apreciar el crecimiento de la CE en los periodos evaluados.

Tabla 1. Caracterización de la CE en búfalos de la raza Murrah desde el destete (10 meses) hasta los 20 meses de edad.

\begin{tabular}{cc}
\hline Rango de edades (meses) & Circunferencia Escrotal(cm) \\
\hline $10-11$ & $12,4 \pm 1,0$ \\
$11-12$ & $13,1 \pm 1,5$ \\
$12-13$ & $14,4 \pm 1,4$ \\
$13-14$ & $14,6 \pm 1,4$ \\
$14-15$ & $14,9 \pm 1,7$ \\
$15-16$ & $16,6 \pm 1,7$ \\
$16-17$ & $17,6 \pm 1,5$ \\
$17-18$ & $18,1 \pm 1,2$ \\
$18-19$ & $19,2 \pm 1,0$ \\
$19-20$ & $20,2 \pm 1,4$ \\
\hline
\end{tabular}

Peso, longitud testicular (derecha - izquierda) y alzada al anca en machos bufalinos. La caracterización de los parámetros morfométricos: peso, longitud testicular (derecha-izquierda) y alzada al anca se presentan en la en la Tabla 2. La figura 2, presenta, el aumento promedio del peso en los periodos evaluados.

Figura 1. Crecimiento de la CE en los periodos evaluados

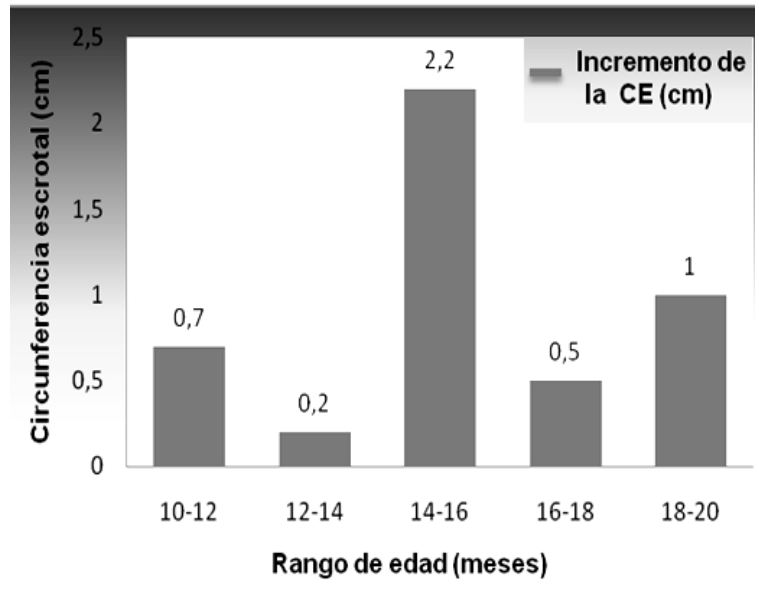

Figura 2. Aumento de peso en los periodos evaluados.

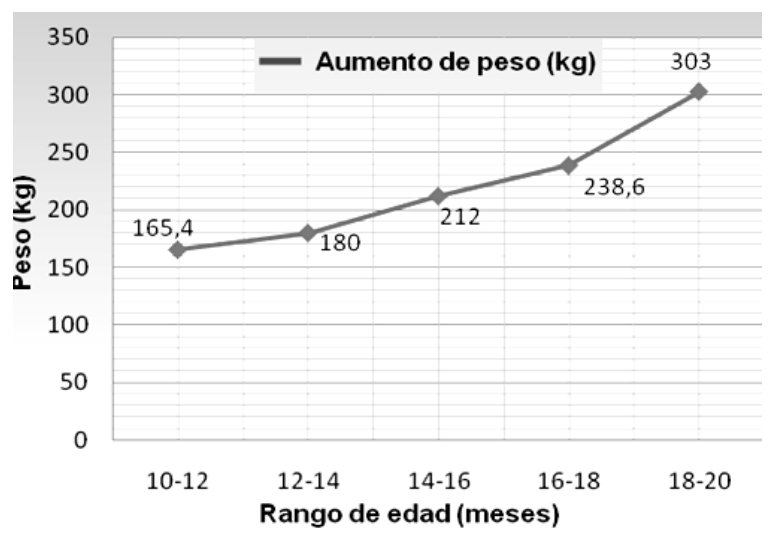

Tabla 2. Peso Corporal, longitud testicular (derecha e izquierda) y alzada al en búfalos machos de la raza Murrah.

\begin{tabular}{|c|c|c|c|c|}
\hline \multirow{2}{*}{ Rango de edades (meses) } & \multirow{2}{*}{ Peso corporal (kg) } & \multicolumn{2}{|c|}{ Longitud testicular (cm) } & \multirow{2}{*}{ Alzada a grupa $(\mathrm{cm})$} \\
\hline & & Derecha & Izquierda & \\
\hline $10-11$ & $162,1 \pm 24,9$ & $4,0 \pm 0,7$ & $4,2 \pm 0,5$ & $104 \pm 4,4$ \\
\hline $11-12$ & $168,7 \pm 24,7$ & $4,3 \pm 0,7$ & $4,4 \pm 0,6$ & $107 \pm 4,1$ \\
\hline $12-13$ & $177,1 \pm 27,5$ & $4,5 \pm 0,7$ & $4,7 \pm 0,6$ & $109 \pm 4,3$ \\
\hline $13-14$ & $182,9 \pm 26,1$ & $4,9 \pm 0,7$ & $4,5 \pm 0,6$ & $110 \pm 4,3$ \\
\hline $14-15$ & $199,4 \pm 28,9$ & $5,2 \pm 0,8$ & $5,2 \pm 0,8$ & $111 \pm 4,1$ \\
\hline $15-16$ & $225,1 \pm 35,1$ & $5,8 \pm 0,9$ & $5,5 \pm 0,8$ & $112 \pm 3,9$ \\
\hline $16-17$ & $230,1 \pm 32,8$ & $6,1 \pm 0,8$ & $5,8 \pm 0,8$ & $114 \pm 4,4$ \\
\hline $17-18$ & $247,1 \pm 29,4$ & $6,4 \pm 0,7$ & $6,2 \pm 0,7$ & $115 \pm 3,6$ \\
\hline $18-19$ & $282,3 \pm 32,1$ & $7,0 \pm 0,6$ & $6,6 \pm 0,6$ & $117 \pm 3,3$ \\
\hline $19-20$ & $323,8 \pm 39,8$ & $7,4 \pm 0,7$ & $6,9 \pm 0,6$ & $118 \pm 3,4$ \\
\hline
\end{tabular}


El Incremento promedio de la alzada al anca a lo largo del estudio se puede apreciar en la figura 3. La tabla 3 , muestra las correlaciones existentes entre CE con los parámetros morfométricos evaluados: edad, peso, longitud testicular (derecha - izquierda) y alzada al anca.

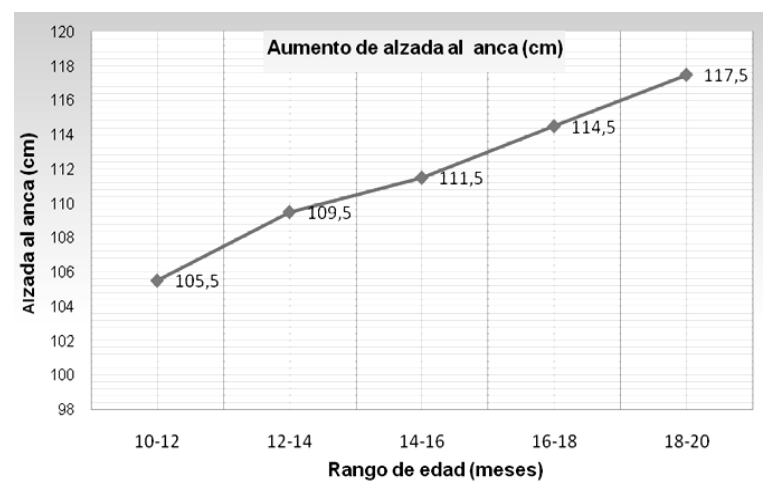

Figura 3. Aumento de alzada al anca en los periodos evaluados.

Tabla 3. Correlación entre circunferencia escrotal, edad, peso, longitud testicular (derecha - izquierda) y alzada al anca para machos bufalinos de la raza Murrah.

\begin{tabular}{cccc}
\hline Parámetro & $\mathbf{n}$ & $\mathbf{r}$ & $\mathbf{R}^{\mathbf{2}}$ \\
\hline CE-Edad & 140 & $0,84^{\star *}$ & 0,92 \\
CE-Peso & 140 & $0,90^{\star *}$ & 0,90 \\
CE-LTD & 140 & $0,93^{\star *}$ & 0,93 \\
CE-LTI & 140 & $0,91^{\star *}$ & 0,92 \\
CE-AG & 140 & $0,71^{* *}$ & 0,95 \\
\hline
\end{tabular}

$\overline{\text { LTD (Longitud testicular derecha); LTI (Longitud testicular izquierda); AG (Alzada }}$ a grupa $){ }^{* \star}(\mathrm{P}<0,01)$

\section{Discusión}

Circunferencia escrotal. Los valores de CE estuvieron entre $12,4 \pm 1,0 \mathrm{~cm}$ y $20,2 \pm 1,4 \mathrm{~cm}$, en el inicio y final del periodo evaluado, presentándose un aumento con valor promedio total de $7,8 \mathrm{~cm}$. Resultados similares han sido reportados por ECHEVERRI (2006), en el mismo grupo racial, con CE de 14,6 y 20,2 cm a los 12 y 22 meses de edad respectivamente. Al comparar los valores medios de la CE observados en el presente estudio con los obtenidos por CRUDELI et al. (2007) y CRUDELI y KONRAD (2013) en la raza Mediterránea, se encontró, que los animales de raza Mediterránea presentaron mayores valores de CE $(21,5 \mathrm{~cm})$, en el rango de edad entre 18 - 22 meses, lo que podría ser explicado, por la oferta nutricional ofrecido a esos animales. Según VALE et al. (2004), en la raza Murrah, la CE ideal para los sementales con edades entre 30 y 36 está alrededor de $30 \pm 3,6 \mathrm{~cm}$, habiendo un crecimiento lineal y correlación positiva $(r>0,90)$ con el peso corporal y la edad del animal.
Al relacionar la $C E$ de los búfalos con resultados obtenidos en machos vacunos, se evidencian grandes diferencias; los vacunos presentan valores de CE similares a edades menores o iguales a 15 meses (media de $30 \mathrm{~cm}$ ), entre los 19 a 21 el valor promedio de CE presentado, esta alrededor $32 \mathrm{~cm}$ (SPITZER, 2000).Por el contrario, los búfalos según este estudio presentan valores de CE más pequeño que lo machos vacunos, obteniéndose valores que oscilan entre 14.4 $\mathrm{cm}$ y $20.2 \mathrm{~cm}$, entre los 15 y 20 meses de Eda. Este mismo comportamiento, fue registrado por Da LUZ, et al., 2013.

La caracterización de la CE mostró que el crecimiento de esta variable no fue uniforme, debido a que, el desarrollo de la CE no siempre va acompañado de las etapas de mayor ganancia de peso. Algunos autores (MOLINA, et al., 1992; BLASCO, 2004; CRUDELI y KONRAD, 2013), han postulado que en mamíferos, en la mayoría de los casos es difícil plantear una ley de crecimiento, debido a factores genéticos, nutricionales y ambientales que pueden hacer variar el peso de los animales y la velocidad de crecimiento.

En la figura1 se presenta el crecimiento de la CE en los periodos evaluados, en donde el menor valor es de $0,2 \mathrm{~cm}$ entre los 12 a 14 meses y el mayor incremento se obtuvo en el periodo de 14 a 16 meses siendo este de $2,2 \mathrm{~cm}$. Se infiere que el crecimiento de este órgano no fue exponencial, es decir no tuvo un incremento constante, como lo han sugerido algunos autores (WITTMAN, 2005). No obstante, este mismo autor y otros investigadores (PIRES et al., 2000) han afirmado que el crecimiento de determinados órganos, como los huesos y músculos se pueden seguir en función del equilibrio del nitrógeno, del calcio y del fósforo, es decir si hay gran aporte de estos minerales se tendrá una mayor tasa de crecimiento. Los órganos tampoco presentan un crecimiento homogéneo, cada uno crece a velocidades distintas, depende entre otras cosas de cuando se necesite la función de dicho órgano durante la ontogénesis. Es decir para el caso del incremento en el tamaño testicular se aplican los principios arriba descritos.

Peso. En general, durante el periodo evaluado el grupo de animales en estudio presentó una ganancia de peso de $161,7 \mathrm{~kg}$, con ganancia diaria de $531,9 \mathrm{~g}$, desde el destete hasta los 20 meses de edad.

Al iniciar la fase experimental, 10 meses de edad, el grupo de animales en estudio, presentó peso promedio de $162,1 \pm 24,9 \mathrm{~kg}$. El peso promedio fue de $165,4 \mathrm{~kg}$, con ganancia diaria de 0,109 gr/día. Durante este periodo los animales se encontraban en un terreno de topografía ondulada, baja oferta forrajera y poca disponibilidad de 
agua. Los factores antes mencionados, probablemente influyeron en el peso corporal, cuando comparados con otro trabajo realizado en Colombia, por ECHEVERRI (2006), quién encontró que el promedio de peso en las razas Murrah, a los 12 y 22 meses de edad, fueron de 157 y 366 kg respectivamente.

Para el periodo comprendido entre 12 a 14 meses los animales en estudio, tuvieron un peso promedio de $180 \mathrm{~kg}$, con ganancias de 5,8kg. La ganancia diaria de peso, disminuyendo con relación al muestreo (95,4 g/días). Varios factores pueden explicar estos eventos, dentro de los cuales, se pueden destacar el estrés del destete (ARANGO, 1992; ARANGO et al., 1992) y/o factores ambientales, sanitarios, manejo y alimentación entre otros (AGUDELO, 2004). Ha denotarse, que durante el predestete en estos sistemas, la alimentación del bucerro depende de la finalidad con que se concibe la crianza del búfalo. Si las condiciones son favorables para el desarrollo de un sistema mixto; producción de carne, leche y venta de reproductores, los animales jóvenes reciben una mejor alimentación. Por el contrario, si las condiciones son propicias para la producción de leche y sus derivados, los bucerros machos recibirán un tratamiento alimentario inferior (NASCIMENTO y MOURA, 1993; LÓPEZ et al., 2007).

Para el periodo entre 14 a 16 meses los animales mostraron un peso promedio de $212 \mathrm{~kg}$, con ganancias promedio de $25,7 \mathrm{~kg}$ para este muestreo, obteniendo $422,7 \mathrm{~g} /$ día en la ganancia diaria de peso, cabe anotar que una de las posibles razones de aumento de peso en este muestreo, está directamente relacionada con los niveles de agua de los humedales, los cuales empezaron a bajar su volumen, beneficiando a los búfalos, en mayor y mejor calidad oferta alimenticia. Los animales en estudio para los 16 a 18 meses mostraron un peso promedio de 238,6 kg, con ganancias de peso de $17 \mathrm{~kg}$ bimensual, y un promedio de ganancia diaria de peso de 279,6 g/dia, disminuyendo esta con relación al muestreo anterior; uno de los factores influyentes en este descenso de peso, está relacionado con la entrada de los animales experimentales a potreros con baja oferta forrajera y alta carga animal, pasando de una carga de 0,66 animales/ha a 1,2 animales/ ha, lo que se considera una carga alta (CERVANTES, 2010).

Entre los 18 a 20 meses, se observó un aumento acelerado en cuanto a peso corporal, alcanzando un promedio de $303 \mathrm{~kg}$, con ganancia bimensual de 41,5 kg. Igualmente, con elevadas ganancias de peso diario de 682,6g/día, estos altos incrementos posiblemente se alcanzaron por la mayor disponibilidad en la oferta forrajera de pastos naturales predominantes debido a las primeras lluvias del año. Por tanto, el presentes resultados contrastan con los encontrados por Da LUZ, et al., (2013), quienes reportaron $446,70 \pm 32,56 \mathrm{~kg}$ de peso corporal en promedio en la raza Murrah, para un rango de edad entre 18 a 24 meses.
Longitud testicular derecha e izquierda. La tabla 2 , muestra la evolución del crecimiento de la longitud testicular. El valor medio de la longitud testicular derecha, osciló entre 4,0 $\pm 0,7$ y $7,4 \pm 0,7 \mathrm{~cm}$, con incrementos por periodos, que fluctuaron entre 0,1 a $0,6 \mathrm{~cm}$. Fueron encontrados valores medios de 4,2 \pm 0,5 y $6,9 \pm 0,6 \mathrm{~cm}$ para la longitud testicular izquierda. El incremento testicular durante los periodos evaluados se mantuvo en el rango entre $-0,2$ a $0,4 \mathrm{~cm}$. Valores similares de longitud testicular fueron relatados por CRUDELI Y KONRAD (2013) y Da LUZ, et al., (2013), en la raza Mediterránea y Murrah respectivamente.

La alzada al anca. En la figura 3 se observa la evolución que este parámetro a lo largo del estudio. Al inicio el estudio mostró un promedio de $104 \pm 4,4 \mathrm{~cm}$ de altura $\mathrm{y}$ al terminar $118 \pm 3,4 \mathrm{~cm}$; indicando un aumento en el periodo experimental de $14 \mathrm{~cm}$, mostrando que el desarrollo en el periodo estudiado fue poco. Además, permite inferir que los animales evaluados, mostraron un crecimiento mayor en la etapa de amamantamiento, con valores medios cercanos a los $100 \mathrm{~cm}$ de altura, lo que evidencia un retraso severo tal vez causado por estrés del destete. En Colombia, ECHEVERRI (2006) relató datos para alzada al anca en machos bufalinos (12 a 20 meses de edad) de la raza Murrah de 107,5 $\mathrm{cm}$ y $128 \mathrm{~cm}$ respectivamente, mientras que en este trabajo los valores obtenidos fueron: $109 \mathrm{~cm}$ y $118 \mathrm{~cm}$. Por otro lados, la investigación de CRUDELI Y KONRAD (2013), en la raza Mediterránea, logró determinar una alzada al anca de $122,6 \mathrm{~cm}$ a una edad comprendida entre 18 a 20 meses

Correlaciones. Las correlaciones entre CE y edad son altas $(r>0,84)$, el modelo de regresión que más se ajusta es lineal; observándose resultados similares en estudios realizados por CUMMING (1999), quien hace referencia que la CE también ha sido positivamente correlacionada con la edad a la pubertad de la progenie.

La CE y el peso están correlacionados positivamente $(r>0,90)$, el modelo de regresión que más se ajusta es logarítmico, estudio realizado por VALE et al., (2004) y Da LUZ, et al., (2013), manifiestan, que así como en los bovinos, en los bufalinos existe una amplia correlación entre el peso corporal y la CE ( $r=0,92 ; 0$, 85 respectivamente).

El valor obtenidos de la correlaciones entre CE y alzada al anca $(r>0,71)$, es considerado alto y positivo, el modelo de regresión que más se ajusta a esa relación es el modelo lineal.

Correlaciones alta y positivamente entre la CE y la longitud testicular (Derecha $r=0,93$; Izquierda $r=0,91$ ), comprueban que la circunferencia de los testículos es 
una medida importante para la evaluación testicular por tanto, es una característica importante para la selección de reproductores bufalinos (Da LUZ, et al., (2013), indicando que puede complementarse con el uso de medidas zoometrícas. El modelo de regresión que más se ajustó a describir el comportamiento de la variable LTD, fue el Lineal, y a LTI fue cuadrático.

Los valores de correlaciones entre CE y los parámetros morfometricos evaluados, muestran altas correlaciones, iguales hallazgos han sido reportados en otras investigaciones realizadas en vacunos y ovinos (MORRYS et al., 1989; RODRÍGUEZ et al., 2013; HOLGADO Y ORTEGA, 2015).

En conclusión, los indicadores estudiados aportan valores sobre el desarrollo corporal y testicular del búfalo macho, de la raza Murrah, criados bajo condiciones de pastoreo natural, lo que contribuye a determinar en un futuro el biotipo requerido para los sistemas productivos locales.

\section{Referencias}

AHMAD, N.; UMAIR, S.; SHAHAB, M.; ARSLAN, M. 2010. Testicular development and establishment of spermatogenesis in Nili-Ravi buffalo bulls. Theriogenology, 73(1):20-25.

AGUDELO, D. 2004. Curvas de crecimiento de crías de vacuno levantadas en la Corporación Universitaria Lasallista. Revista Lasallista de Investigación.1 (2): 42 - 45. Disponible en: http://www.redalyc.org/articulo.oa?id=69510207. Consultado (12/08/2016).

ALBADRY, K. I.; JAAFER ZALZALA, S.; IBRAHIM, F. F.; HURISH, K. F. 2016. Monthly and Seasonality Variation in Freezability of Semen and Associations Between Freezability of Semen With Age and Testicular Dimension of Holstein Bulls Born in Iraq. PARIPEX-Indian Journal of Research, 5(1): 35-38.

ARANGO, J. 1992. Crecimiento predestete en becerros de vacas Brahman y toros Brahman, Guzerá y Nelor en Apure. Trabajo de Ascenso. Universidad Central de Venezuela, Facultad de Ciencias Veterinarias. Maracay, Venezuela. Disponible en: http://www.revfacagronluz.org.ve/v15 3/v153z008.html. Consultado (10/10 /2016).

ARANGO, J.; PLASSE, D.; VERDE, O.; HOOGESTEIJN, R.; FOSSI, R.; BASTIDAS, P.; RODRÍGUEZ, R. 1992. Peso al destete en becerros Bos indicus y toros Brahman, Guzerá y Nelor en Apure. En: Resúmenes del VII Congreso Venezolano de Zootecnia. GR-28. Disponible en: http://www.revfacagronluz.org.ve/v15 3/v153z008.html. Consultado $(10 / 10 / 2016)$.

CERVANTES, E. 2010. Caracterización de parámetros productivos, reproductivos y contables de una explotación de ganado bufalino en Hacienda Mosquito, San Marcos, Sucre. Trabajo de grado para optar el título de Zootecnista. Programa de Zootecnia, Facultad de Ciencias Agropecuarias. Universidad de Sucre, Sincelejo. 53 Pág.

BOURDON, R. y BRINKS, J. 1986. Scrotal circumference in yearling Hereford bulls: adjustment factors, heritabilities and genetic, environmental and phenotypic relationships with growth traits. J. Anim. Sci. 62 (4), 958-967.

BLASCO, A. 2004. XIV Curso internacional sobre mejora genética animal. Universidad Politécnica de Valencia. (Consultado el 1 de septiembre de 2016). En: http://redalyc.uaemex.mx/pdf/695/69510207.pdf. Consultado (10/10 12016).

CORBET N. J.; BURNS B. M.; JOHNSTON D. J.; WOLCOTT M. L.; CORBET D. H.; VENUS B. K.; LI Y.; MCGOWAN M. R.; HOLROYD R. G. 2012. Male traits and herd reproductive capability in tropical beef cattle. 2. Genetic parameters of bull traits. Animal Production Science, 53: 101-113.

CRUDELI, G. A.; KONRAD, J. L. 2013. Evaluación del crecimiento en hembras y machos bubalinos en Argentina. Revista Brasileira de Reprodução Animal, 37 (2): 115-120. 
CRUDELI, G.; POCHON, D.; OLAZARRI, M.; MONZÓN, N.; CHAPARRO, L.; FLORES, S.; PATIÑO, E.; CEDRÉS, J. 2007. Morphometric evaluation of male Mediterranean buffaloes in Northern Corrientes, Argentina. VIII World Buffalo Congres Disponible en: http://ijas.pagepress.org/index.php/ijas/article/viewFile/ijas.2007.s2.1281/1209. Consultado (22/10/2016).

CUMMING, B. 1999. Bull soundness-reproduction. News Agriculture. 12 (4) 935-939.

Da LUZ, P. A. C.; ANDRIGHETTO, C.; SANTOS, P. R. S.; JORGE, A., CONSTANTINO, M. V. P., PEREIRA, F. T. V., MESS, A. NETO, A. C. A. 2012. Daily sperm production and evaluation of morphological reproductive parameters of Murrah buffaloes in an extensive breeding system. Spermatogenesis, 2(2), 88-93.

Da LUZ, P. A. C.; SANTOS, P. R. da S.; ANDRIGHETTO, C., JORGE; A. M.; De ASSIS NETO, A. C. 2013. The Correlation between Age, Body Weight and Testicular Parameters in Murrah Buffalo Bulls Raised in Brazil.The Journal of Reproduction and Development,59(1): 14-17.

ECHEVERRI, J. 2006. Bufalometría en butoretes en la hacienda la Suiza, de propiedad del Fondo Ganadero del Centro, localizada en el Magdalena Medio antioqueño. Memorias III Simposio de Búfalos de la Américas. Pág. 32-39.

GOOGLE EARTH. 2016. (Consultado: el 30 de Agosto del 2016). En: http://earth.google.es/intl/es_es/thanks.html \#os=win\#chrome=yes\#updater=yes Consultado $(10 / 10 / 2016)$.

HOLGADO, F. D.; ORTEGA, M. F. 2015. Caracterización de toros adultos de la raza criollo argentino: peso corporal, alzada y circunferencia escrotal. Actas Iberoamericanas de Conservación Animal AICA, 6: 172-177.

IGAG. 2010. Estudio general de suelos y zonificación de tierras del Departamento de Sucre.

IRANO N.; De CAMARGO G.M.F.; COSTA R. B.; TERAKADO, A.P. N.; MAGALHÃES A.F.B.; SILVA RMDO, et al. 2016. Genome-Wide Association Study for Indicator Traits of Sexual Precocity in Nellore Cattle. PLoS ONE 11(8): e0159502. doi:10.1371/journal.pone.0159502

MATEOS, E. 1992. Estudio de los parámetros de valoración del rendimiento reproductivo en macho cabrío de las razas Verata y Malagueña. Tesis Doctoral. Universidad Complutense de Madrid. Facultad de Veterinaria. Departamento de Patología Animal II. Madrid, España. http://biblioteca.ucm.es/tesis/19911996/D/2/AD2008901.pdf (Consultado el 3 de agosto de 2010).

MOLINA, A.; SERRANO, M. I.; JIMÉNEZ, J. M.; SALADO, F. M.; CABEZA de VACA, F.; ESPARRÁGO, E.; RODERO, A. 1992. Estimación de la curva de crecimiento en ganado retinto: Aspectos prácticos para la tipificación de pesos. Arch. Zootec, 41 (extra): $543-548$.

MORRYS, L.; TYNER, P.; MORRIS, L.; FORGASON, L.; WILLIAMS, S.; YOUNG, F. 1989. Correlation between scrotal circumference and age in American Brahman Bulls. Theriogenology, 31: 489-491.

NASCIMENTO, C.; MOURA, L. 1993. Crianza de Búfalos: Alimentación, manejo e instalaciones. EMBRAPA, Brasil, 10 Pág.

NEMA, S.; KODAGALI, S. 1994. Trans - scrotal circumference (TSC), age, body weight and seminal character in surti bulls. Indian Journal of Animal Reproduction, 15 (2): 154-156.

LÓPEZ, J.; FUNDORA, O.; FLORIO, J. 2007. Sistema de Alimentación y manejo de bucerros. Rev. Producción y Negocio 4: 20 -28.

PÉREZ - OSORIO, JAIR.; CHACÓN - JARAMILLO, LILIANA.; OTERO - ARROYO, RAFAEL JOSÉ; CARDONA ÁLVAREZ, JOSÉ.; ANDRADE SOUZA, FERNANDO. 2014. Relación entre la circunferencia escrotal, el crecimiento testicular y parámetros de calidad de semen en toros de raza Guzerat, desde la pubertad hasta los 36 meses de edad. Revista de Medicina Veterinaria, (27): 73-87. 
PIRES, C.; SILVA, L. F.; FARINATTI, L. H.; PEIXOTO, L. A.; FÜLBER, M. E.; CUNHA, M.A. 2000. Crescimento de cordeiros abatidos com diferentes pesos. 2. Constituintes corporais. Ciência Rural 30 (5): 869-873.

RAMíREZ-LÓPEZ, C.; RUGELES-PINTO, C.; GUIMARAES, J. D.; VERGARA-GARAY, O. 2016. Relación entre biometría testicular y circunferencia escrotal en toretes de la raza Nelore en Brasil. Revista Científica, 26(1): 49-54.

RODRÍGueZ, F. D. J. B.; CORDERO, M. A. O.; HERNÁNDEZ, G. T.; CEDILLO, F. D. J. M.; CAMACHO, J. M. G.; GÓMEZ, M. O. D. 2013. Relación de la edad, peso corporal y medidas morfométricas sobre el inicio de la pubertad en corderos polypay del altiplano potosino. Revista Científica, 23(5).

SAMPAIO I.B.M. Estatística aplicada à experimentação animal. 2a ed. Belo Horizonte: FEP-MVZ; 2002. p. 265.

SILVA, M. R.; PEDROSA, V.B.; SILVA, J. B. C.; HERRERA, L. G. G.; ELER, J. P.; ALBUQUERQUE, L. G. 2012. Parámetros genéticos de las características andrológicas en la especie bovina. Arquivos de Medicina Veterinária, 44(1): 1-11.

SPITZER, J. C. 2000. Bull breeding soundness evaluation: Current status. In: Topics in Bull Fertility, P. J. Chenoweth, ed. International Veterinary Information System. Ithaca, NY.

TERAKADO, A. P. N.; A. A. BOLIGON, F. BALDI, J. A. I. V. SILVA, AND L. G. ALBUQUERQUE. 2015. Genetic associations between scrotal circumference and female reproductive traits in Nelore cattle1. J. Anim. Sci. 93:27062713.

VALE, W; MAGALHÃES, N; MAGALHÃES, D y RIBEIRO, H. 2004. Testis growth, body weight and testicular size rates in the Brazilian murrah buffaloes $X^{\text {th }}$ Int Cong Anim Reprod Porto Seguro: 1,179-pp

VALE, W. G. 2011. Avances biotecnológicos en reproducción de búfalos. Revista Tecnología en Marcha, 24(5):89-104.

WITTMAN, J. 2005. En: Fisiología Veterinaria. Editores: Engelhardt, W, y Breves G. Editorial Acribia, S.A. Zaragoza España, Pág. 473-475. 